(C) by Japan Society for Cell Biology

\title{
A Replica Method Useful in the Isolation of Mutants of FM3A, a Non-Adherent Cell Line from Mouse Mammary Carcinoma
}

\author{
Hiroshi Matsuzaki, Masatake Yamauchi ${ }^{1}$ and Isao Shibuya \\ Department of Biochemistry, Saitama University, Urawa 338, Japan
}

\begin{abstract}
Existing replica methods designed for adherent somatic cells are not applicable to non-adherent cells of tumor origin. We have developed a new, efficient replica method for the negative selection of mutants of nonadherent mouse FM3A cells. Mutagenized cells are grown clonally on soft-agar plates then allowed to proliferate upwards through layered polyester disks, thus producing replicas with the same colony patterns. Isolation of mutants with increased requirements for myo-inositol by this method is described.
\end{abstract}

Isolation of somatic cell mutants has become increasingly important in the genetics and biochemistry of mammalian cells, but convenient methods for mutant isolation are not available for all types of cell lines. Mutants with defects in essential genes are, in general, difficult to isolate; they are expected to have phenotypes of conditional lethality, including auxotrophy, that only are detected negatively by a replica method among the many wild-type cells. Concentration procedures $(5,10)$ facilitate isolation in such negative selections, but an efficient replica-preparing step is highly desirable.

Cohesive somatic cells, such as Chinese hamster ovary (CHO) cells in monolayer can be replicated from a plastic surface to filter paper (4). Raetz et al. (9) further improved this method by using layered disks of polyester sheets through which the CHO cells proliferate upwards producing disks with the same colony patterns. The growth characteristics and surface properties of most non-adherent cells of tumor origin, however, differ from those of adherent $\mathrm{CHO}$ cells. In fact, Raetz et al. found that some myelomas and hybridomas penetrated only one polyester cloth layer with plating efficiencies of $40-80 \%$ (9).

We examined the applicability of these methods to mouse FM3A, a cell line established from a spontaneous mammary carcinoma in a $\mathrm{C} 3 \mathrm{H} / \mathrm{He}$ mouse (8) and found that none worked satisfactorily. A coat of poly-L-lysine (9) helped the adsorption of FM3A cells on the plastic dishes, but no clear clonal growth was observed on the dishes or on stacked polyester disks. FM3A cells, typical non-adherent cells that grow while floating, offer many advantages as a model cell line in somatic cell biology; they have low heteroploidy (11), relatively high growth rates (8), a low requirement for fetal bovine serum (8), high efficiencies of colony formation on soft-agar media (7) and high frequencies of DNA-mediated transformations (3). Lack of an efficient replica method for FM3A cells, however, has limited the isolation of mutants mainly

1 Present address: Institute of Applied Microbiology, University of Tokyo, Bunkyo-ku, Tokyo 113, Japan 
to direct selections, such as drug resistance $(1,6)$. We describe here a new replica method for FM3A cells that combines the convenient properties of polyester cloth and the ability of FM3A cells to grow as clones on soft-agar media.

Mouse FM3A, subclone F28-7 cells (1) were cultured routinely in suspension at $37^{\circ} \mathrm{C}$ in ES medium (6, supplied by Nissui Seiyaku Co., Tokyo, Japan) supplemented with $2 \%$ fetal bovine serum (Gibco, Grand Island, N.Y.). To isolate auxotrophic mutants we used a synthetic ES' medium supplemented with $2 \%$ dialyzed fetal bovine serum with, or without, the nutrient to be tested. The ES' medium was identical to ES medium except that it lacked the test nutrient. Fetal bovine serum first was dialyzed according to the method of Koyama and Kodama (6), then dialyzed against 10 volumes of ES' medium. The soft-agar plates used contained ES or ES' medium supplemented with 5\% dialyzed fetal bovine serum and $0.5 \%$ agar (Purified Agar, Difco Laboratories, Detroit, Mich.). All the media used contained $100 \mu \mathrm{g} / \mathrm{ml}$ of

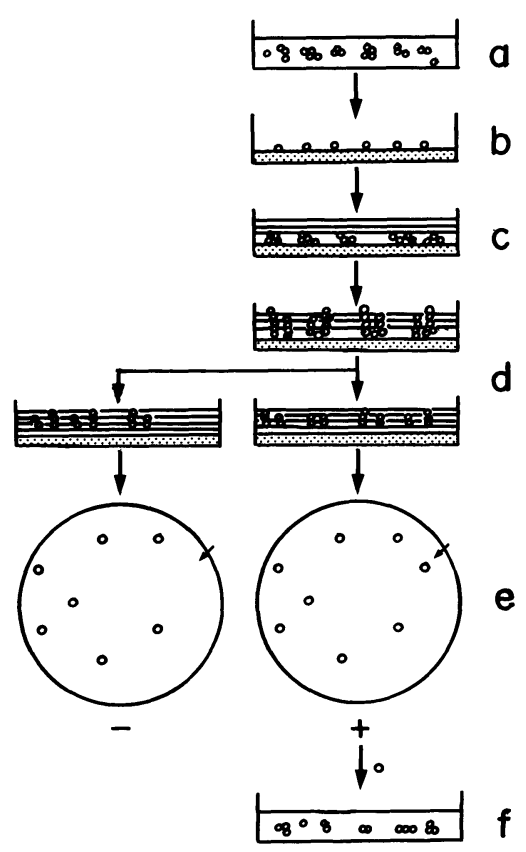

Fig. 1. Isolation of mutants of non-adherent mouse FM3A cells by the polyester disk replica method. Mutagenized cells (a) are plated on soft-agar medium at 500-1,000 cells per plate then incubated under conditions permissive for isolation of the mutant (b). After incubation (e.g., for 7 days at $37^{\circ} \mathrm{C}$ ) three polyester disks are layered carefully on the agar and thoroughly wetted with fresh medium so as to leave no bubbles between the disks and agar surface (c). After further incubation (d), the middle and bottom disks are transferred separately onto fresh polyester disks (one sheet each) placed on soft-agar plates with compositions permissive $(\mathrm{d},+)$ and nonpermissive $(\mathrm{d},-)$ to the mutant, if an auxotrophic mutant is wanted. Two new polyester disks are overlaid on these disks, then incubation under permissive $(\mathrm{d},+)$ and selective $(\mathrm{d},-)$ conditions is done to prepare the second-round replicas (d). The second disks from the top in both dishes are stained and compared for mutant clones (e). The third disk from the top is incubated under permissive conditions thus serving as the master from which individual mutant candidates can be isolated by mixing them with a piece of fresh agar medium using a small spoon then transferring them to medium for suspension culture (f). 
streptomycin sulfate and $100 U / \mathrm{ml}$ of penicillin G. Log-phase cells $\left(2 \times 10^{5}\right.$ cells $\left./ \mathrm{ml}\right)$ were mutagenized for $4 \mathrm{~h}$ at $37^{\circ} \mathrm{C}$ in culture medium with $500 \mathrm{ng} / \mathrm{ml}$ of $N$-methyl- $N^{\prime}$ nitro- $N$-nitrosoguanidine. Auxotrophic mutants were concentrated, prior to replica selection, by 5-bromodeoxyuridine treatment as described elsewhere (5). Polyester disks $82 \mathrm{~mm}$ in diameter, which fitted the culture dishes snugly, were cut from PE20 sheets (mesh opening $20 \mu \mathrm{m}$, Swiss Silk Bolting Cloth Mfg. Co., Ltd., Thal, Switzerland) with a circular burin designed for this purpose. The disks were washed, then sterilized and stained with Coomassie brilliant blue G-250, and recycled as described by Raetz et al. (9).

The replica method developed for the negative selection of mutants, as well as for the simultaneous examination of phenotypes of many clones derived from mouse FM3A cells is given in Fig. 1. Both the plating efficiency of FM3A cells on soft-agar media (Fig. 1, step b) and on polyester disks from agar (Fig. 1, step c) usually was $100 \%$, irrespective of the number of colonies plated per dish. A typical set of three polyester disks, which satisfactorily gave identical colony patterns, is shown in Fig. 2. The pattern on the top disk usually is not clear (as Raetz et al. found for CHO cells [9]) and this top disk we discarded. Examination of polyester sheets with mesh openings ranging from 5 to $92 \mu \mathrm{m}$ revealed that the sheet with the $20 \mu \mathrm{m}$ opening gives the best proliferation of FM3A cells and the clearest colony patterns.

Plating efficiencies in the second-round replica preparation (Fig. 1, step d) depended on the density of the colonies; efficiencies were almost $100 \%$ when the first-round replica disks held 100 or less colonies, whereas efficiencies were lower with more colonies per disk. From polyester disks with about 1,000 colonies, we obtained second-round replica disks that harbored clear colonies ranging from 800 to 900 . But, even in such cases, the colony patterns obtained under the two conditions (Fig. 1, step e) were essentially the same, suggesting that common small colonies were lost during the second-round transfer (step d). It was possible to identify mutant candidates by comparing the two stained disks (step e). It was useful to place a new polyester disk between the soft-agar and the first-round replica disk for better efficiency (Fig. 1, step d).

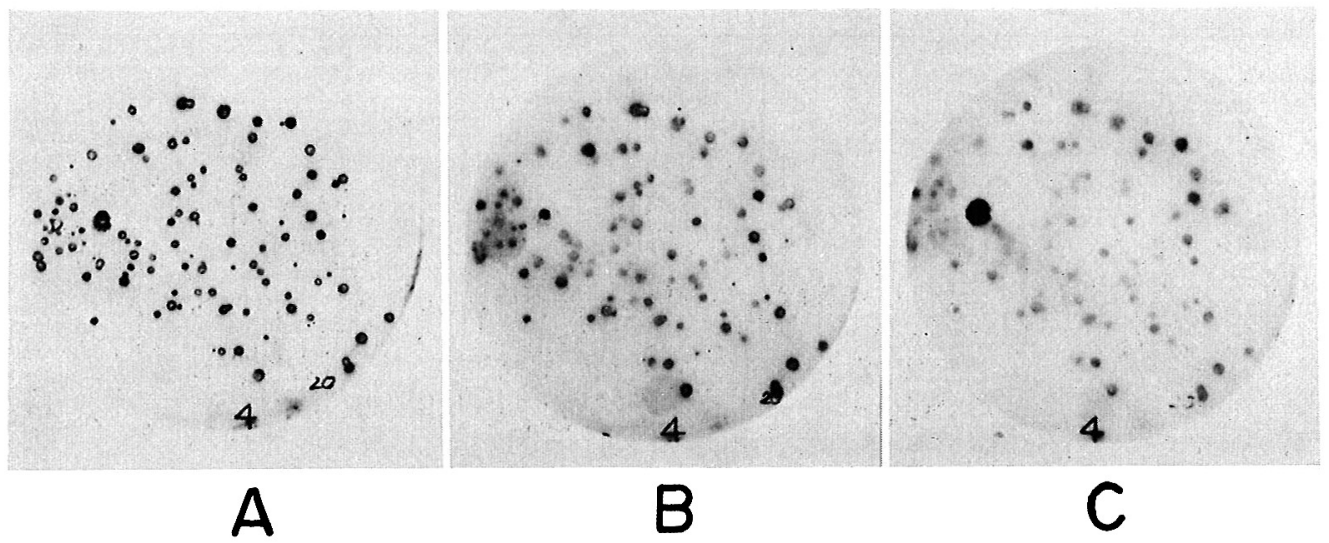

Fig. 2. Three stained polyester disks showing the same colony patterns. Wild-type FM3A cells were allowed to grow as clones on ES-agar medium at $37^{\circ} \mathrm{C}$ for 7 days, then three layers of polyester disks with mesh opening of $20 \mu \mathrm{m}$ were overlaid and the dish incubated for 7 days. All disks were stained with Coomassie blue. A, the bottom polyester disk; B, the middle disk; C, the top disk. 


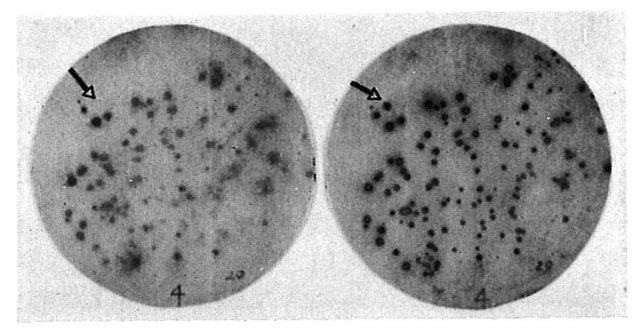

A

B

Fig. 3. Detection of an FM3A mutant with an increased myo-inositol requirement by a comparison of replica disks incubated with $10 \mu \mathrm{M}$ (A) and $1 \mathrm{mM}$ (B) myo-inositol. The second disks from the top in the second-round replica preparation (see Fig. 1) were stained with Coomassie blue. Arrows indicate the presence (B) and the absence (A) of a candidate myo-inositol mutant colony. The corresponding portion of the master disk can be transferred to suspension culture to examine growth characteristics in detail.

Using this replica method, we have been able to isolate several different types of FM3A auxotrophic mutants. As an example, we describe here the isolation of mutants with increased requirements for myo-inositol. Approximately $1 \times 10^{5}$ mutagenized cells were cultured at $37^{\circ} \mathrm{C}$ for 5 days in medium supplemented with $1 \mathrm{mM}$ myoinositol for fixation. These then were starved for $24 \mathrm{~h}$ for excess myo-inositol in

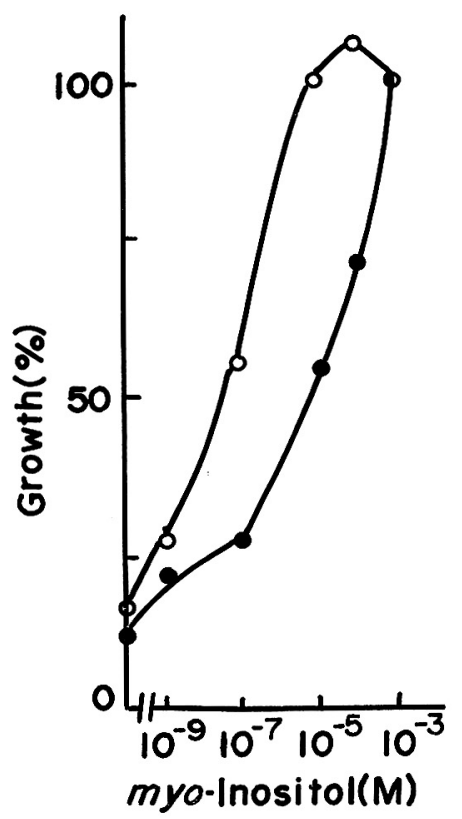

Fig. 4. Effect of myo-inositol on the growth of wild-type (F28-7, O) and mutant (MI50, •) cells. Cells seeded at $1 \times 10^{5}$ per plate in ES'-dialyzed serum were starved of myo-inositol for $24 \mathrm{~h}$, then the medium was replaced with ES'-dialyzed serum containing various concentrations of myo-inositol. After 3 days of incubation at $37^{\circ} \mathrm{C}$, cell numbers were counted with a hemacytometer, and expressed as percents of the values of each strain cultured with $1 \mathrm{mM}$ myo-inositol. Errors in duplicate cultures were $<10 \%$ of each value. 
ES' medium supplemented with dialyzed serum and $10 \mu \mathrm{M}$ myo-inositol. 5Bromodeoxyuridine treatment concentrated the mutant by a factor of 5. Approximately 10,000 cells were seeded on 20 agar plates, then replica selection of mutant candidates that grew with $1 \mathrm{mM} m y o$-inositol but did not grow with $10 \mu \mathrm{M}$ myoinositol was made. Plating efficiency in the replica formation of the first round was $100 \%$ and of the second-round $85 \%$. From 16 candidates isolated, two mutants with increased requirements for myo-inositol were obtained. The detection of a mutant with stained polyester disks is shown in Fig. 3, and the effect of myo-inositol concentration on the growth of wild-type cells and one of the mutants (strain MI50) is shown in Fig. 4. Compounds related structurally and metabolically to myo-inositol (phosphatidylinositol, phosphatidic acid, D-mannitol, and glycerol) did not restore the growth defect of strain MI50, evidence of a specific requirement of the mutant for myo-inositol.

The replica method described here is sufficiently simple and efficient that it should be applicable, in principle, to the isolation of other types of mutant FM3A cells (e.g., temperature-sensitive mutants and mutants with altered cell surface characteristics) and those of other cell lines of tumor origin with similar properties.

Acknowledgments. We thank Drs. T. Seno, D. Ayusawa, and H. Koyama for the mouse FM3A cells and their valuable discussions on our research, and Dr. M. Nishijima for his advice on the handling of the polyester cloth. This study was supported in part by Grant-in-Aid for Scientific Research and for Cancer Research from the Ministry of Education, Science and Culture of Japan.

\section{REFERENCES}

1. Ayusawa, D., K. Iwata and T. Seno. Alteration of ribonucleotide-reductase in aphidicolinresistant mutants of mouse FM3A cells with associated resistance to arabinosyladenine and arabinosylcytosine. Somat. Cell Genet. 7, 27-42, 1981

2. Ayusawa, D., H. Koyama, K. Iwata and T. Seno. Selection of mammalian thymidine auxotrophic cell mutants defective in thymidylate synthase by their reduced sensitivity to methotrexate. Somat. Cell Genet. 7, 523-534, 1981

3. Ayusawa, D., K. Shimizu, H. Koyama, K. Takeishi and T. Seno. Unusual aspects of human thymidylate synthase in mouse cells introduced by DNA-mediated gene transfer. J. Biol. Chem. 258, 48-53, 1983

4. Esko, J.D., and C.R.H. RaETz. Replica plating and in situ enzymatic assay of animal cell colonies established on filter paper. Proc. Natl. Acad. Sci. U.S.A. 75, 1190-1193, 1978

5. KAO, F.T. and T.T. PUCK. Genetics of somatic mammalian cells. VII. Induction and isolation of nutritional mutants in Chinese hamster cells. Proc. Natl. Acad. Sci. U.S.A. 60, 1275-1281, 1968

6. Koyama, H. and H. Kodama. Adenine phosphoribosyltransferase deficiency in cultured mouse mammary tumor FM3A cells resistant to 4-carbamoylimidazolium 5-olate. Cancer Res. 42, 4210-5214, 1982

7. Kuroki, T. Colony formation of mammalian cells on agar plates and its application to Lederberg's replica plating. Exp. Cell Res. 80, 55-62, 1973

8. NAKANO, N. Establishment of cell lines in vitro from a mammary ascites tumor of mouse and biological properties of the established lines in a serum containing medium. Tohoku J. Exp. Med. 88, 69-84, 1966

9. Raetz, C.R.H., M.M. Wermuth, T.M. McIntyre, J.D. Esko and D.C. Wing. Somatic cell cloning in polyester stacks. Proc. Natl. Acad. Sci. U.S.A. 79, 3223-3227, 1982

10. Thompson, L.H., J.L. Harkins and C.P. Stanners. A mammalian cell mutant with a temperature-sensitive leucyl-transfer RNA synthetase. Proc. Natl. Acad. Sci. U.S.A. 70, 3094-3098, 
1973

11. Yoshida, M.C. Q-banded karyotype of a mouse FM3A cell line. Chromosomal Information Service 32, 31-32, 1982

(Received for publication, October 28, 1985) 